\title{
Deriving Macroscopic Myocardial Conductivities by Homogenization of Microscopic Models
}

\author{
Paul E. Hand*, Boyce E. Griffith, Charles S. Peskin \\ Courant Institute of Mathematical Sciences, New York University, New York, NY, USA
}

Received: 16 September 2008 / Accepted: 2 April 2009 / Published online: 2 May 2009

(C) Society for Mathematical Biology 2009

\begin{abstract}
We derive the values for the intracellular and extracellular conductivities needed for bidomain simulations of cardiac electrophysiology using homogenization of partial differential equations. In our model, cardiac myocytes are rectangular prisms and gap junctions appear in a distributed manner as flux boundary conditions for Laplace's equation. Using directly measurable microproperties such as cellular dimensions and endto-end and side-to-side gap junction coupling strengths, we inexpensively obtain effective conductivities close to those given by simulations with a detailed cyto-architecture (Stinstra et al. in Ann. Biomed. Eng. 33:1743-1751, 2005). This model provides a convenient framework for studying the effect on conductivities of aligned vs. brick-like arrangements of cells and the effect of different distributions of gap junctions along the myocyte membranes.
\end{abstract}

Keywords Homogenization · Bidomain - Syncytium · Effective conductivity · Cardiac modeling

\section{Introduction}

Computer simulations have the potential to increase our understanding of normal and pathological cardiac function, and to improve the effectiveness of clinical therapies. Although the biophysics at the cellular level is well understood, whole heart simulations that resolve every cell are computationally infeasible at present. Instead, a more tractable approach is to perform macroscopic simulations based on the bidomain equations (Henriquez, 1993), which govern locally averaged potentials inside and outside cells. These equations require physical values of the effective conductivity of intracellular and extracellular regions in both the longitudinal (fiber) and the transverse (cross-fiber) directions. Ideally, such values should be directly obtained from measurable cellular properties, such as geometry and gap junctional conductivity. Otherwise, the values are free parameters which must be chosen by matching simulation results to experiments.

\footnotetext{
* Corresponding author.

E-mail addresses: hand@ cims.nyu.edu (Paul E. Hand), griffith@ cims.nyu.edu (Boyce E. Griffith), peskin@cims.nyu.edu (Charles S. Peskin).
} 
There are relatively few studies which attempt to derive the macroscopic parameters required by the bidomain equations directly from measurable microscale quantities. One approach to obtain these values is homogenization of partial differential equations (PDEs) as in Neu and Krassowska (1993). In this approach, the intracellular region of tissue is modeled as a collection of periodically arranged cells connected through physical openings that correspond to gap junctions. They can compute effective conductivities by solving Laplace's equation on a periodic domain. Although these authors present a detailed derivation involving homogenization, they resort to a resistor network model in order to find analytical formulae for the effective bidomain conductivities. A shared inconvenience of their PDE and resistor network models is that they intertwine transverse and longitudinal gap junctional connections, making it difficult to assign proper values based on separate experimental measurements of side-to-side and end-to-end coupling strengths (Yao et al., 2003).

An alternative approach to computing passive conductivities is given by Stinstra et al. (2005), who create a detailed tissue model designed to account for realistically complex cell shapes with random variability. Stinstra et al. then solve Laplace's equation over a domain containing several cells to obtain the effective conductivities. Although their simulations involve a high level of detail in the cellular microstructure, their calculation for intracellular transverse conductivity yields values an order of magnitude less than those in the experimental literature. They propose two possible explanations of this discrepancy: The total gap junctional conductivity may be larger than measured, and the gap junctions may be more preferentially located on the cell sides than is measured. In the present paper, we explore the feasibility of both explanations.

In this paper, we follow a homogenization approach similar to that of Neu and Krassowska (1993) to derive the effective conductivities of cardiac tissue. Unlike this earlier homogenization work, however, we employ our microscale PDE model to obtain the macroscopic bidomain parameters. In particular, we do not resort to a resistor network to obtain the macroscopic parameters from the measured microscale quantities. In our approach, we separate the structure and placement of cells from the gap junctional connections between them. Specifically, we idealize cells as rectangular prisms, inside of which the electric potential satisfies Laplace's equation. Instead of modeling gap junctions as discrete geometrical entities akin to Fig. 1(a), we include their effect as boundary conditions on each cell membrane as in Fig. 1(b). This approach assumes no more detail than is provided by direct measurements, such as those of Yao et al. (2003). These modeling decisions make a mathematically natural framework within which to study the effects on conductivity of the arrangement of cells and of the distribution of gap junctions on cell membranes. In some cases, we are able to obtain analytical formulae for the effective conductivities. In cases where we cannot do so, we need only to solve Laplace's equation on one cell of fixed geometry.

The remainder of this paper proceeds as follows: In Section 2, we motivate the calculation of effective conductivities by considering their role in the bidomain equations. Section 3 sets up the electrostatic Laplace's equation in the aligned cellular arrangement. In Section 4, we perform the homogenization in the aligned arrangement and calculate the numerical values of the effective conductivities. Similarly, in Section 5, we formulate the electrostatic problem in a brick-like cellular arrangement, perform the homogenization, and calculate the corresponding conductivities. In Section 6, we compare our conductivity values to those in the literature and discuss the relevance of our computations to 
a

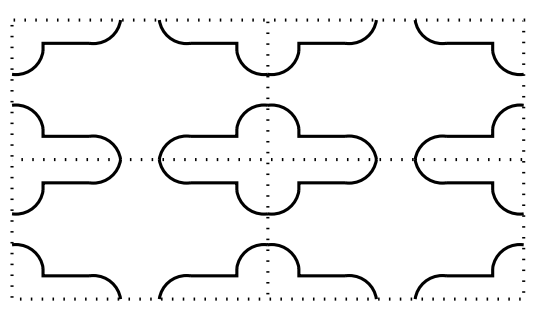

$\mathrm{b}$

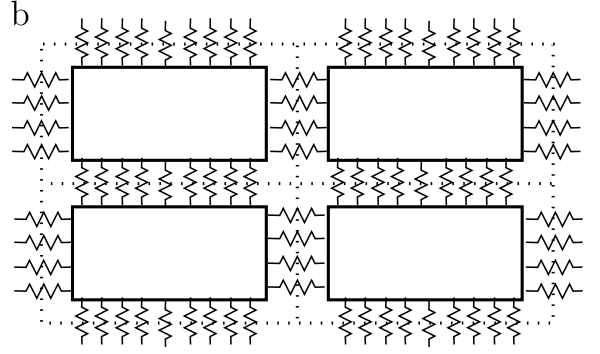

Fig. 1 Instead of modeling gap junctions through complex cellular geometry (a), we model them through flux boundary conditions on simple geometry (b). The multiple resistors shown in (b) represent a continuous boundary condition, see (2)-(7).

electromechanical simulations. In Section 7, we present our conclusions. In Appendix A, we present additional details regarding the derivation of the extracellular effective conductivities. In Appendix B, we present the numerical values for the physical parameters that enter our cellular model.

\section{Bidomain equations and effective conductivity}

The bidomain equations provide the most realistic macroscopic description of the electrical activity of cardiac tissue under normal and pathological conditions (Henriquez, 1993). They govern the intracellular and extracellular electric potential in an averaged sense:

$$
\begin{aligned}
& \nabla \cdot\left(\sigma_{\mathrm{i}} \nabla \phi_{\mathrm{i}}^{0}\right)=\beta\left(C \partial_{t}\left(\phi_{\mathrm{i}}^{0}-\phi_{\mathrm{e}}^{0}\right)+I_{\text {ion }}\left(\phi_{\mathrm{i}}^{0}-\phi_{\mathrm{e}}^{0}, \omega\right)\right), \\
& \nabla \cdot\left(\sigma_{\mathrm{e}} \nabla \phi_{\mathrm{e}}^{0}\right)=-\beta\left(C \partial_{t}\left(\phi_{\mathrm{i}}^{0}-\phi_{\mathrm{e}}^{0}\right)+I_{\text {ion }}\left(\phi_{\mathrm{i}}^{0}-\phi_{\mathrm{e}}^{0}, \omega\right)\right),
\end{aligned}
$$

where $\sigma_{\mathrm{i}}$ and $\sigma_{\mathrm{e}}$ are the macroscopic conductivity tensors of the intracellular and extracellular spaces, $\beta$ is the membrane surface area per unit volume of tissue, $C$ is the membrane capacitance per unit area, $\omega$ stands for relevant gating variables, and $I_{\text {ion }}$ is the ionic current per unit area of the membrane. Here, $\phi_{\mathrm{i}}^{0}(t, \vec{x})$ and $\phi_{\mathrm{e}}^{0}(t, \vec{x})$ are each defined for all $\vec{x}$, and are to be interpreted as the locally averaged intracellular or extracellular potential near the point $\vec{x}$. Note that the intracellular and extracellular potential are each separately averaged, not averaged with each other.

We write the superscript ' 0 ' because $\phi_{\mathrm{i}}^{0}$ is the leading order term of the asymptotic expansion (15) for intracellular potential. Similarly, $\phi_{\mathrm{e}}^{0}$ is the leading order term of a corresponding expansion for extracellular potential. If we locally align the coordinates with the myocardial fibers so that the $x_{1}$ direction coincides with the fiber direction and assume that the $x_{2}$ and $x_{3}$ directions are locally indistinguishable, then the conductivity tensors are diagonal and involve only the longitudinal and transverse conductivities:

$$
\sigma_{\mathrm{i}}=\left(\begin{array}{ccc}
\sigma_{\mathrm{i}, 1} & 0 & 0 \\
0 & \sigma_{\mathrm{i}, \mathrm{t}} & 0 \\
0 & 0 & \sigma_{\mathrm{i}, \mathrm{t}}
\end{array}\right) \quad \text { and } \quad \sigma_{\mathrm{e}}=\left(\begin{array}{ccc}
\sigma_{\mathrm{e}, 1} & 0 & 0 \\
0 & \sigma_{\mathrm{e}, \mathrm{t}} & 0 \\
0 & 0 & \sigma_{\mathrm{e}, \mathrm{t}}
\end{array}\right) \text {. }
$$



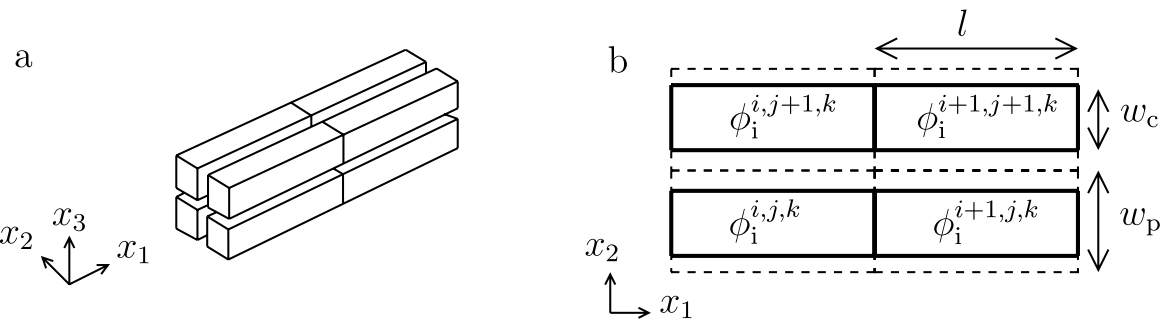

Fig. 2 The aligned cellular architecture: (a) cells of dimension $l \times w_{\mathrm{c}} \times w_{\mathrm{c}}$ are arranged in three-space with period $l \times w_{\mathrm{p}} \times w_{\mathrm{p}}$; (b) an $x_{1}, x_{2}$ cross-section.

A goal of the present work is to determine $\sigma_{\mathrm{i}, 1}, \sigma_{\mathrm{i}, \mathrm{t}}, \sigma_{\mathrm{e}, 1}, \sigma_{\mathrm{e}, \mathrm{t}}$ directly from measured microscopic quantities.

\section{Full cellular model in an aligned arrangement}

We model myocytes as $l \times w_{\mathrm{c}} \times w_{\mathrm{c}}$ prisms arranged periodically in three-space with period $l \times w_{\mathrm{p}} \times w_{\mathrm{p}}$, see Fig. 2 . The length of the cells $l$ is assumed to equal the longitudinal period, but the width and height of the cells $w_{\mathrm{c}}$ are smaller than the transverse periods $w_{\mathrm{p}}$ in order to provide an extracellular volume fraction $\alpha=1-\left(w_{\mathrm{c}} / w_{\mathrm{p}}\right)^{2}$. In this model, the intracellular space is not physically contiguous. Instead, resistive connections allow current to flow directly between the interiors of adjacent cells. Note that we do not attempt to account for the branching of cells in the present work. Additionally, we ignore fiber rotation because our analysis is purely local.

To identify the bidomain parameters $\sigma_{\mathrm{i}}$ and $\sigma_{\mathrm{e}}$, we consider the degenerate case of a steady state without transmembrane ionic current. In this situation, the equations for intracellular and extracellular potential decouple. In the following, we consider only the intracellular potential; the similar calculations for the extracellular potential are described in Appendix A.

The electric potential in the intracellular space satisfies Laplace's equation

$$
-\Delta \phi_{\mathrm{i}}^{i, j, k}(\vec{x})=0 \quad \text { in } \Omega_{\mathrm{i}}^{i, j, k},
$$

where $\Omega_{\mathrm{i}}^{i, j, k}=[i \cdot l,(i+1) \cdot l] \times\left[j \cdot w_{\mathrm{p}}, j \cdot w_{\mathrm{p}}+w_{\mathrm{c}}\right] \times\left[k \cdot w_{\mathrm{p}}, k \cdot w_{\mathrm{p}}+w_{\mathrm{c}}\right]$ is the region occupied by the $(i, j, k)$ th cell, and $\phi_{\mathrm{i}}^{i, j, k}$ is the intracellular potential of the $(i, j, k)$ th cell, indicated in Fig. 2(b). For ease of notation, we identify the domain of the function $\phi_{\mathrm{i}}^{i, j, k}$ with $[0, l] \times\left[0, w_{\mathrm{c}}\right] \times\left[0, w_{\mathrm{c}}\right]$. We denote position by $\vec{x}=\left(x_{1}, x_{2}, x_{3}\right)$.

We model the gap junctions between cells in a continuous manner through boundary conditions on (1). The current density between two neighboring cells is proportional to the potential difference between the positions on opposite sides of the gap junctions: 


$$
\begin{aligned}
-\sigma_{\mathrm{c}} \partial_{x_{1}} \phi_{\mathrm{i}}^{i, j, k}\left(l, x_{2}, x_{3}\right) & =\frac{g_{\mathrm{GJ}, \text { end }}}{w_{\mathrm{c}}^{2}}\left(\phi_{\mathrm{i}}^{i, j, k}\left(l, x_{2}, x_{3}\right)-\phi_{\mathrm{i}}^{i+1, j, k}\left(0, x_{2}, x_{3}\right)\right), \\
-\sigma_{\mathrm{c}} \partial_{x_{2}} \phi_{\mathrm{i}}^{i, j, k}\left(x_{1}, w_{\mathrm{c}}, x_{3}\right) & =\frac{g_{\mathrm{GJ}, \mathrm{side}}}{l \cdot w_{\mathrm{c}}}\left(\phi_{\mathrm{i}}^{i, j, k}\left(x_{1}, w_{\mathrm{c}}, x_{3}\right)-\phi_{\mathrm{i}}^{i, j+1, k}\left(x_{1}, 0, x_{3}\right)\right), \\
-\sigma_{\mathrm{c}} \partial_{x_{3}} \phi_{\mathrm{i}}^{i, j, k}\left(x_{1}, x_{2}, w_{\mathrm{c}}\right) & =\frac{g_{\mathrm{GJ}, \mathrm{side}}}{l \cdot w_{\mathrm{c}}}\left(\phi_{\mathrm{i}}^{i, j, k}\left(x_{1}, x_{2}, w_{\mathrm{c}}\right)-\phi_{\mathrm{i}}^{i, j, k+1}\left(x_{1}, x_{2}, 0\right)\right), \\
-\sigma_{\mathrm{c}} \partial_{x_{1}} \phi_{\mathrm{i}}^{i, j, k}\left(l, x_{2}, x_{3}\right) & =-\sigma_{\mathrm{c}} \partial_{x_{1}} \phi_{\mathrm{i}}^{i+1, j, k}\left(0, x_{2}, x_{3}\right), \\
-\sigma_{\mathrm{c}} \partial_{x_{2}} \phi_{\mathrm{i}}^{i, j, k}\left(x_{1}, w_{\mathrm{c}}, x_{3}\right) & =-\sigma_{\mathrm{c}} \partial_{x_{2}} \phi_{\mathrm{i}}^{i, j+1, k}\left(x_{1}, 0, x_{3}\right), \\
-\sigma_{\mathrm{c}} \partial_{x_{3}} \phi_{\mathrm{i}}^{i, j, k}\left(x_{1}, x_{2}, w_{\mathrm{c}}\right) & =-\sigma_{\mathrm{c}} \partial_{x_{3}} \phi_{\mathrm{i}}^{i, j, k+1}\left(x_{1}, x_{2}, 0\right),
\end{aligned}
$$

where $\sigma_{\mathrm{c}}$ is the cytoplasmic conductivity $(\mathrm{mS} / \mathrm{cm}), g_{\mathrm{GJ}, \text { end }}$ is the total conductance $(\mathrm{mS})$ of all gap junctions on one end of a cell, and $g_{\mathrm{GJ}, \text { side }}$ is the total conductance $(\mathrm{mS})$ of all gap junctions on one side of a cell. Equations (2)-(4) balance cytosolic and gap junctional current, whereas (5)-(7) equate the current leaving each cell with the current entering its appropriate neighbor. See Table B.1 for the physical parameters and variables introduced for this model.

In the remainder of the present section, we nondimensionalize (1)-(7) and state the effective conductivity problem in this aligned cellular arrangement. Note that the model could easily be generalized to allow for more complex geometries, such as the 'jutting' cells analyzed in Hubbard et al. (2007). It could also allow gap junctional density to be varying within the ends or sides of cells. As an illustration, we explore the effects of a brick-like cellular arrangement in Section 5.

\subsection{Nondimensionlization}

We rescale space so that cells are of length $\varepsilon$ :

$$
\tilde{\vec{x}}=\frac{\varepsilon}{l} \vec{x} .
$$

Dropping the tildes, (1)-(7) become

$$
\begin{aligned}
-\Delta \phi_{\mathrm{i}}^{i, j, k}(\vec{x}) & =0 \quad \text { in }[0, \varepsilon] \times\left[0, \varepsilon h_{\mathrm{c}}\right] \times\left[0, \varepsilon h_{\mathrm{c}}\right], \\
-\partial_{x_{1}} \phi_{\mathrm{i}}^{i, j, k}\left(\varepsilon, x_{2}, x_{3}\right) & =\frac{1}{\varepsilon} \kappa_{\text {end }}\left(\phi_{\mathrm{i}}^{i, j, k}\left(\varepsilon, x_{2}, x_{3}\right)-\phi_{\mathrm{i}}^{i+1, j, k}\left(0, x_{2}, x_{3}\right)\right), \\
-\partial_{x_{2}} \phi_{\mathrm{i}}^{i, j, k}\left(x_{1}, \varepsilon h_{\mathrm{c}}, x_{3}\right) & =\frac{1}{\varepsilon} \kappa_{\text {side }}\left(\phi_{\mathrm{i}}^{i, j, k}\left(x_{1}, \varepsilon h_{\mathrm{c}}, x_{3}\right)-\phi_{\mathrm{i}}^{i, j+1, k}\left(x_{1}, 0, x_{3}\right)\right), \\
-\partial_{x_{3}} \phi_{\mathrm{i}}^{i, j, k}\left(x_{1}, x_{2}, \varepsilon h_{\mathrm{c}}\right) & =\frac{1}{\varepsilon} \kappa_{\text {side }}\left(\phi_{\mathrm{i}}^{i, j, k}\left(x_{1}, x_{2}, \varepsilon h_{\mathrm{c}}\right)-\phi_{\mathrm{i}}^{i, j, k+1}\left(x_{1}, x_{2}, 0\right)\right), \\
-\partial_{x_{1}} \phi_{\mathrm{i}}^{i, j, k}\left(\varepsilon, x_{2}, x_{3}\right) & =-\partial_{x_{1}} \phi_{\mathrm{i}}^{i+1, j, k}\left(0, x_{2}, x_{3}\right), \\
-\partial_{x_{2}} \phi_{\mathrm{i}}^{i, j, k}\left(x_{1}, \varepsilon h_{\mathrm{c}}, x_{3}\right) & =-\partial_{x_{2}} \phi_{\mathrm{i}}^{i, j+1, k}\left(x_{1}, 0, x_{3}\right), \\
-\partial_{x_{3}} \phi_{\mathrm{i}}^{i, j, k}\left(x_{1}, x_{2}, \varepsilon h_{\mathrm{c}}\right) & =-\partial_{x_{3}} \phi_{\mathrm{i}}^{i, j, k+1}\left(x_{1}, x_{2}, 0\right),
\end{aligned}
$$


a

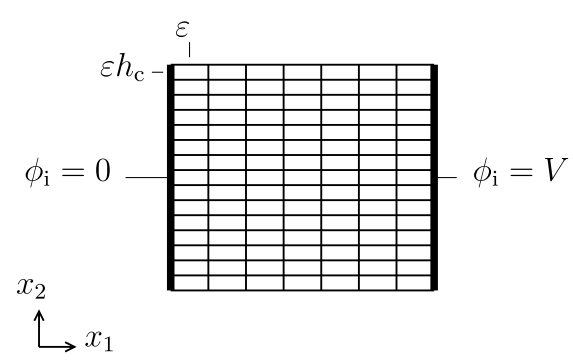

$\mathrm{b}$

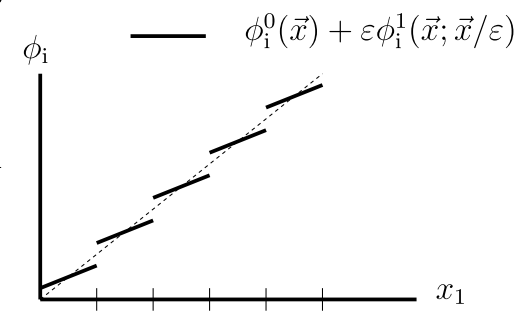

Fig. 3 In order to compute effective conductivity, we impose a potential difference across a large number of cells and calculate the resulting current density (a). In this figure, extracellular space has been omitted for clarity. The resulting electric potential is depicted in (b).

where $\kappa_{\text {end }}=\frac{g_{\mathrm{GJ}} \text {,end } l}{\sigma_{\mathrm{c}} w_{\mathrm{c}}^{2}}$ is the nondimensional end-to-end gap junctional conductance, $\kappa_{\text {side }}=$ $\frac{g_{\mathrm{GJ}} \text { side }}{\sigma_{\mathrm{c}} w_{\mathrm{c}}}$ is the nondimensional side-to-side gap junctional conductance, the intracellular domain of the $(i, j, k)$ th cell is of size $[0, \varepsilon] \times\left[0, \varepsilon h_{\mathrm{c}}\right] \times\left[0, \varepsilon h_{\mathrm{c}}\right]$, and $h_{\mathrm{c}}=w_{\mathrm{c}} / l$. Letting $h_{\mathrm{p}}=w_{\mathrm{p}} / l$, the tissue microstructure now has period $\varepsilon \times \varepsilon h_{\mathrm{p}} \times \varepsilon h_{\mathrm{p}}$. The small parameter $\varepsilon$ can be interpreted as the ratio of the length of a myocyte to the length scale of significant variations of electrical potential within the tissue; see Neu and Krassowska (1993) for further details.

\subsection{Statement of the effective conductivity problem}

Consider a cube of tissue of dimensionless size $1 \times 1 \times 1$, composed of cells of size $\varepsilon \times \varepsilon h_{\mathrm{c}} \times \varepsilon h_{\mathrm{c}}$ arranged with period $\varepsilon \times \varepsilon h_{\mathrm{p}} \times \varepsilon h_{\mathrm{p}}$. The index notation distinguishing the $(i, j, k)$ th cell is cumbersome, and we introduce $\phi_{\mathrm{i}}(\vec{x})$ as a single function defined over $\Omega_{\mathrm{i}}=\bigcup_{i, j, k} \Omega_{\mathrm{i}}^{i, j, k}$. The function $\phi_{\mathrm{i}}$ satisfies the PDE (8) within each cell, and microscopic boundary conditions akin to (9)-(14) on the boundary of each cell. As depicted in Fig. 3(a), it will also satisfy macroscopic boundary conditions:

$$
\begin{aligned}
\phi_{\mathrm{i}}\left(0, x_{2}, x_{3}\right) & =0, \\
\phi_{\mathrm{i}}\left(1, x_{2}, x_{3}\right) & =V, \\
\partial_{x_{2}} \phi_{\mathrm{i}}\left(x_{1}, 0, x_{3}\right) & =0, \\
\partial_{x_{2}} \phi_{\mathrm{i}}\left(x_{1}, 1, x_{3}\right) & =0, \\
\partial_{x_{3}} \phi_{\mathrm{i}}\left(x_{1}, x_{2}, 0\right) & =0, \\
\partial_{x_{3}} \phi_{\mathrm{i}}\left(x_{1}, x_{2}, 1\right) & =0 .
\end{aligned}
$$

The dimensional intracellular longitudinal conductivity is then given by

$$
\sigma_{\mathrm{i}, 1}=\sigma_{c} \cdot \frac{V}{\frac{1}{A} \iint_{\Omega_{\mathrm{i}} \cap\left\{x_{1}=c\right\}} \partial_{x_{1}} \phi_{\mathrm{i}} d x_{2} d x_{3}},
$$


where $A$ is the cross-sectional area of the tissue, the domain of integration is the set of interior points across a cross-section, and the integral is independent of the constant $c$.

Intracellular transverse conductivity can be determined analogously, provided the macroscopic boundary conditions are altered to impose the potential difference $V$ in the $x_{2}$-direction.

\section{Homogenization in an aligned arrangement}

In a homogeneous medium with no microstructure, we would expect the potential to change linearly in the direction of the applied potential difference. Such a $\phi_{\mathrm{i}}$ will not satisfy the cellular boundary conditions, and hence we add a small amplitude correction that varies on the length scale of a cell. We are led to the homogenization ansatz:

$$
\phi_{i}(\vec{x})=\phi_{\mathrm{i}}^{0}(\vec{x})+\varepsilon \phi_{\mathrm{i}}^{1}(\vec{x} ; \vec{x} / \varepsilon)+\cdots,
$$

where $\phi_{\mathrm{i}}^{1}$ is $1 \times h_{\mathrm{p}} \times h_{\mathrm{p}}$ periodic in its second variable and is only defined when that variable, $\vec{x} / \varepsilon$, is in a $1 \times h_{\mathrm{c}} \times h_{\mathrm{c}}$ subregion of that period. We will denote the second variable by $\vec{y}=\left(y_{1}, y_{2}, y_{3}\right)$. Both $\phi_{\mathrm{i}}^{0}$ and $\phi_{\mathrm{i}}^{1}$ are defined for all values of the first variable $\vec{x}$. Note that an equation similar to (15) could be obtained by guessing that the electric potential behaves like $\phi_{\mathrm{i}}^{0}(\vec{x})$ plus a periodic disturbance. We present the homogenization ansatz instead because it parallels the derivation of the bidomain equations in Neu and Krassowska (1993), and also because it is applicable to more general macroscopic boundary conditions. For a more general treatment of homogenization in the context of bidomain equations, see Neu and Krassowska (1993) or Keener and Sneyd (1998).

Applying the ansatz (15) to the PDE (8)-(14) and extracting the leading order terms in $\varepsilon$, we arrive at

$$
\begin{aligned}
& -\Delta_{y} \phi_{\mathrm{i}}^{1}(\vec{x} ; \vec{y})=0 \quad \text { in } Y_{\mathrm{i}}=[0,1] \times\left[0, h_{\mathrm{c}}\right] \times\left[0, h_{\mathrm{c}}\right], \\
& -\partial_{y_{1}} \phi_{\mathrm{i}}^{1}\left(x ; 1, y_{2}, y_{3}\right)-\kappa_{\mathrm{end}}\left(\phi_{\mathrm{i}}^{1}\left(x ; 1, y_{2}, y_{3}\right)-\phi_{\mathrm{i}}^{1}\left(x ; 0, y_{2}, y_{3}\right)\right)=\partial_{x_{1}} \phi_{\mathrm{i}}^{0}(x), \\
& -\partial_{y_{2}} \phi_{\mathrm{i}}^{1}\left(x ; y_{1}, h_{\mathrm{c}}, y_{3}\right)-\kappa_{\text {side }}\left(\phi_{\mathrm{i}}^{1}\left(x ; y_{1}, h_{\mathrm{c}}, y_{3}\right)-\phi_{\mathrm{i}}^{1}\left(x ; y_{1}, 0, y_{3}\right)\right)=\partial_{x_{2}} \phi_{\mathrm{i}}^{0}(x), \\
& -\partial_{y_{3}} \phi_{\mathrm{i}}^{1}\left(x ; y_{1}, y_{2}, h_{\mathrm{c}}\right)-\kappa_{\text {side }}\left(\phi_{\mathrm{i}}^{1}\left(x ; y_{1}, y_{2}, h_{\mathrm{c}}\right)-\phi_{\mathrm{i}}^{1}\left(x ; y_{1}, y_{2}, 0\right)\right)=\partial_{x_{3}} \phi_{\mathrm{i}}^{0}(x), \\
& -\partial_{y_{1}} \phi_{\mathrm{i}}^{1}\left(x ; 0, y_{2}, y_{3}\right)=-\partial_{y_{1}} \phi_{\mathrm{i}}^{1}\left(x ; 1, y_{2}, y_{3}\right), \\
& -\partial_{y_{2}} \phi_{\mathrm{i}}^{1}\left(x ; y_{1}, 0, y_{3}\right)=-\partial_{y_{2}} \phi_{\mathrm{i}}^{1}\left(x ; y_{1}, h_{\mathrm{c}}, y_{3}\right), \\
& -\partial_{y_{3}} \phi_{\mathrm{i}}^{1}\left(x ; y_{1}, y_{2}, 0\right)=-\partial_{y_{3}} \phi_{\mathrm{i}}^{1}\left(x ; y_{1}, y_{2}, h_{\mathrm{c}}\right),
\end{aligned}
$$

where $\Delta_{y}$ is the Laplacian in the variable $\vec{y}=\left(y_{1}, y_{2}, y_{3}\right)$. By linearity, $\phi_{\mathrm{i}}^{1}$ can be expressed in terms of $\phi_{\mathrm{i}}^{0}$

$$
\phi_{\mathrm{i}}^{1}(\vec{x} ; \vec{y})=\sum_{k=1}^{3} \partial_{x_{k}} \phi_{\mathrm{i}}^{0}(\vec{x}) w_{k}(\vec{y}),
$$




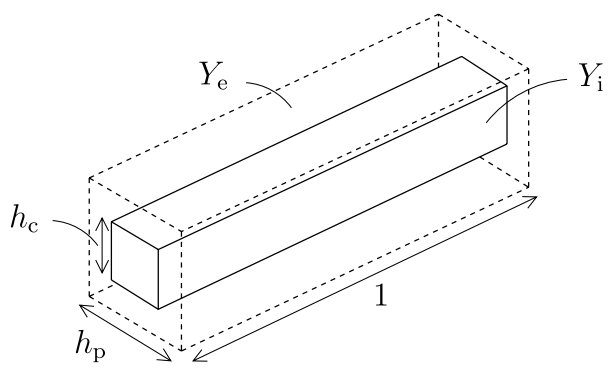

Fig. 4 The unit cell in the aligned geometry is composed of an intracellular region $Y_{\mathrm{i}}$ and an extracellular region $Y_{\mathrm{e}}$.

where $w_{k}$ solves the corrector problem

$$
\begin{aligned}
& -\Delta_{y} w_{k}(\vec{y})=0 \quad \text { in }[0,1] \times\left[0, h_{\mathrm{c}}\right] \times\left[0, h_{\mathrm{c}}\right], \\
& -\partial_{y_{1}} w_{k}\left(1, y_{2}, y_{3}\right)-\kappa_{\text {end }}\left(w_{k}\left(1, y_{2}, y_{3}\right)-w_{k}\left(0, y_{2}, y_{3}\right)\right)=\delta_{k 1}, \\
& -\partial_{y_{2}} w_{k}\left(y_{1}, h_{\mathrm{c}}, y_{3}\right)-\kappa_{\text {side }}\left(w_{k}\left(y_{1}, h_{\mathrm{c}}, y_{3}\right)-w_{k}\left(y_{1}, 0, y_{3}\right)\right)=\delta_{k 2}, \\
& -\partial_{y_{3}} w_{k}\left(y_{1}, y_{2}, h_{\mathrm{c}}\right)-\kappa_{\text {side }}\left(w_{k}\left(y_{1}, y_{2}, h_{\mathrm{c}}\right)-w_{k}\left(y_{1}, y_{2}, 0\right)\right)=\delta_{k 3}, \\
& -\partial_{y_{1}} w_{k}\left(0, y_{2}, y_{3}\right)=-\partial_{y_{1}} w_{k}\left(1, y_{2}, y_{3}\right), \\
& -\partial_{y_{2}} w_{k}\left(y_{1}, 0, y_{3}\right)=-\partial_{y_{2}} w_{k}\left(y_{1}, h_{\mathrm{c}}, y_{3}\right), \\
& -\partial_{y_{3}} w_{k}\left(y_{1}, y_{2}, 0\right)=-\partial_{y_{2}} w_{k}\left(y_{1}, y_{2}, h_{\mathrm{c}}\right) .
\end{aligned}
$$

Here, $\delta_{k j}=1$ if $k=j$, and 0 otherwise. Notice that finding each of the $w_{k}$ only requires solving Laplace's equation over one cell, depicted in Fig. 4.

We can compute the effective conductivities in the longitudinal and transverse directions by applying a potential and determining the average current density. For a voltage applied in the $x_{1}$-direction, the nondimensional current within cells is

$$
-\partial_{x_{1}} \phi_{\mathrm{i}}(x)=-\partial_{x_{1}} \phi_{\mathrm{i}}^{0}(x)-\partial_{y_{1}} \phi_{\mathrm{i}}^{1}(x ; x / \varepsilon)+O(\varepsilon) .
$$

The average longitudinal current density across a period near $\vec{x}$ is thus

$$
i_{\text {avg }}=-\partial_{x_{1}} \phi_{\mathrm{i}}^{0}(x) \frac{1}{h_{\mathrm{p}}^{2}} \int_{0}^{h_{\mathrm{c}}} \int_{0}^{h_{\mathrm{c}}}\left(1+\partial_{y_{1}} w_{1}\left(y_{1}, y_{2}, y_{3}\right)\right) d y_{2} d y_{3},
$$

which is independent of $y_{1}$. Hence, the effective conductivity in an aligned cell arrangement is

$$
\sigma_{\mathrm{i}, \mathrm{l}}=\frac{\sigma_{\mathrm{c}}}{h_{\mathrm{p}}^{2}} \int_{0}^{h_{\mathrm{c}}} \int_{0}^{h_{\mathrm{c}}}\left(1+\partial_{y_{1}} w_{1}\left(y_{1}, y_{2}, y_{3}\right)\right) d y_{2} d y_{3} .
$$

Similarly, the transverse conductivity in an aligned cell arrangement is

$$
\sigma_{\mathrm{i}, \mathrm{t}}=\frac{\sigma_{\mathrm{c}}}{1 \cdot h_{\mathrm{p}}} \int_{0}^{h_{\mathrm{c}}} \int_{0}^{1}\left(1+\partial_{y_{2}} w_{2}\left(y_{1}, y_{2}, y_{3}\right)\right) d y_{1} d y_{3} .
$$




\subsection{Analytical solution to the corrector problem}

Equations (16)-(22), which define the corrector problems for aligned geometry, can be solved exactly. The problems are effectively one-dimensional and their solutions are

$$
\begin{aligned}
& w_{1}(\vec{y})=\frac{1}{1+\kappa_{\text {end }}}\left(\frac{1}{2}-y_{1}\right), \\
& w_{2}(\vec{y})=\frac{1}{1+\kappa_{\text {side }} h}\left(\frac{h}{2}-y_{2}\right), \\
& w_{3}(\vec{y})=\frac{1}{1+\kappa_{\text {side }} h}\left(\frac{h}{2}-y_{3}\right),
\end{aligned}
$$

of which the first is depicted in Fig. 3(b).

\subsection{Resulting effective conductivities}

The effective longitudinal and transverse conductivities derived from (23)-(27) are

$$
\begin{aligned}
& \sigma_{\mathrm{i}, 1}=\sigma_{\mathrm{c}}(1-\alpha)\left(1-\frac{1}{1+\kappa_{\text {end }}}\right), \\
& \sigma_{\mathrm{i}, \mathrm{t}}=\sigma_{\mathrm{c}} \sqrt{1-\alpha}\left(1-\frac{1}{1+\kappa_{\text {side }} h}\right) .
\end{aligned}
$$

Using the cellular parameters in Table B.1, these become

$$
\begin{aligned}
\sigma_{\mathrm{i}, 1} & =1.01 \mathrm{mS} / \mathrm{cm}, \\
\sigma_{\mathrm{i}, \mathrm{t}} & =0.03 \mathrm{mS} / \mathrm{cm} .
\end{aligned}
$$

\subsection{Equivalent resistor network}

For the aligned arrangement, the effective conductivities in the longitudinal and transverse directions could be more easily computed by considering the cytoplasms and gap junctions as two resistors in series. For example, the total cytosolic longitudinal resistance for a tissue of length $L$ and cross-sectional area $A$ is $\frac{1}{\sigma_{\mathrm{c}}} \frac{L}{(1-\alpha) A}$. The total resistance of gap junctions is $\frac{L}{\ell}\left(\frac{1}{g_{\mathrm{GJ}, \text { end }}} \frac{w_{\mathrm{p}}^{2}}{A}\right)$. Combining these in series and computing the effective conductivity, one can obtain (28). A similar calculation applies for obtaining (29). No similar simplification will end up providing such an immediate calculation of longitudinal and transverse conductivities for the brick-like arrangement we detail next.

\section{Full cellular model and homogenization in a brick-like arrangement}

The histology of the myocardium shows that cells in adjacent fibers are not aligned. We model this by assuming a half-cell offset between neighboring fibers, resulting in the brick-like arrangement shown in Fig. 5. 

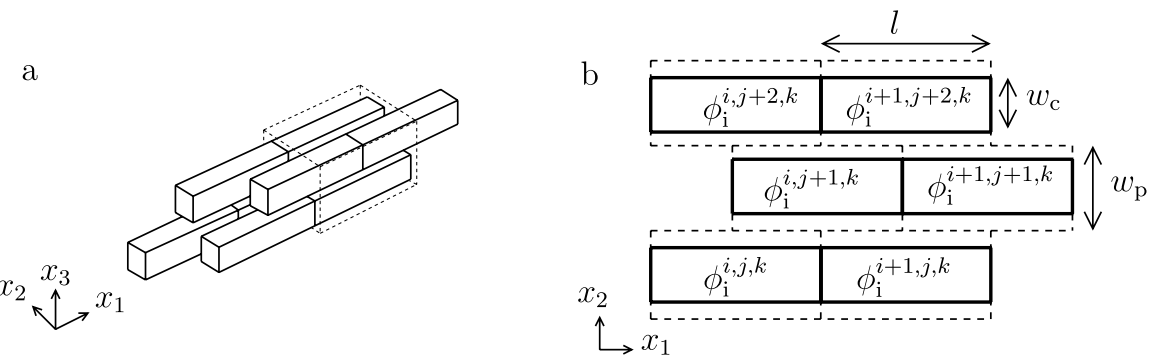

Fig. 5 The brick-like cellular architecture. Cells of dimension $l \times w_{\mathrm{c}} \times w_{\mathrm{c}}$ are arranged with a period of $l \times w_{\mathrm{p}} \times w_{\mathrm{p}}$, except that adjacent fibers are offset by half a cell length (a). The arrangement can be viewed as the periodic extension of the region inside the dashed prism. An $x_{1}, x_{2}$ cross-section is shown in (b).

The nondimensional analogs of (8)-(14) are tedious to write down because each cell in brick geometry is adjacent to 10 cells. Using the notation 'nbh' to denote the correct neighbor to the $(i, j, k)$ th cell, (8)-(14) become

$$
\begin{aligned}
-\Delta \phi_{\mathrm{i}}^{i, j, k}(\vec{x}) & =0 \\
-\sigma_{\mathrm{c}} \partial_{x_{1}} \phi_{\mathrm{i}}^{i, j, k}\left(1, x_{2}, x_{3}\right) & =\frac{g_{\mathrm{GJ}, \mathrm{end}}}{w_{\mathrm{c}}^{2}}\left(\phi_{\mathrm{i}}^{i, j, k}\left(1, x_{2}, x_{3}\right)-\phi_{\mathrm{i}}^{\mathrm{nbh}}\left(0, x_{2}, x_{3}\right)\right), \\
-\sigma_{\mathrm{c}} \partial_{x_{2}} \phi_{\mathrm{i}}^{i, j, k}\left(x_{1}, h_{\mathrm{c}}, x_{3}\right) & =\frac{g_{\mathrm{GJ}, \text { side }}}{l w_{\mathrm{c}}}\left(\phi_{\mathrm{i}}^{i, j, k}\left(x_{1}, h_{\mathrm{c}}, x_{3}\right)-\phi_{\mathrm{i}}^{\mathrm{nbh}}\left(x_{1}+1 / 2,0, x_{3}\right)\right), \\
-\sigma_{\mathrm{c}} \partial_{x_{3}} \phi_{\mathrm{i}}^{i, j, k}\left(x_{1}, x_{2}, h_{\mathrm{c}}\right) & =\frac{g_{\mathrm{GJ}, \mathrm{side}}}{l w_{\mathrm{c}}}\left(\phi_{\mathrm{i}}^{i, j, k}\left(x_{1}, x_{2}, h_{\mathrm{c}}\right)-\phi_{\mathrm{i}}^{\mathrm{nbh}}\left(x_{1}+1 / 2, x_{2}, 0\right)\right), \\
-\sigma_{\mathrm{c}} \partial_{x_{1}} \phi_{\mathrm{i}}^{i, j, k}\left(1, x_{2}, x_{3}\right) & =-\sigma_{\mathrm{c}} \partial_{x_{1}} \phi_{\mathrm{i}}^{\mathrm{nbh}}\left(0, x_{2}, x_{3}\right), \\
-\sigma_{\mathrm{c}} \partial_{x_{2}} \phi_{\mathrm{i}}^{i, j, k}\left(x_{1}, h_{\mathrm{c}}, x_{3}\right) & =-\sigma_{\mathrm{c}} \partial_{x_{2}} \phi_{\mathrm{i}}^{\mathrm{nbh}}\left(x_{1}+1 / 2,0, x_{3}\right), \\
-\sigma_{\mathrm{c}} \partial_{x_{3}} \phi_{\mathrm{i}}^{i, j, k}\left(x_{1}, x_{2}, h_{\mathrm{c}}\right) & =-\sigma_{\mathrm{c}} \partial_{x_{3}} \phi_{\mathrm{i}}^{\mathrm{nbh}}\left(x_{1}+1 / 2, x_{2}, 0\right) .
\end{aligned}
$$

Notice that the boundary conditions in the transverse directions, $y_{2}$ and $y_{3}$, involve the values of $\phi_{\mathrm{i}}$ at the neighboring cell, offset by half a cell length. As in Section 3, we rescale space so that each cell is of length $\varepsilon$ and consider a single function $\phi_{\mathrm{i}}(\vec{x})$ defined over the interiors of all cells. The ansatz is the same as (15):

$$
\phi_{i}(\vec{x})=\phi_{\mathrm{i}}^{0}(\vec{x})+\varepsilon \phi_{\mathrm{i}}^{1}(\vec{x} ; \vec{x} / \varepsilon)+\cdots,
$$

but now $\phi_{\mathrm{i}}^{1}$ is periodic in its second variable with period $\varepsilon \times 2 \varepsilon h_{\mathrm{p}} \times 2 \varepsilon h_{\mathrm{p}}$. This period is depicted by the dashed prism in Fig. 5(a). As before,

$$
\phi_{\mathrm{i}}^{1}(\vec{x} ; \vec{y})=\sum_{k=1}^{3} \partial_{x_{k}} \phi_{\mathrm{i}}^{0}(\vec{x}) w_{k}(\vec{y}) .
$$


Due to a symmetry between the four cells within a period of $w_{k}$,

$$
\begin{aligned}
w_{k}\left(y_{1}+1, y_{2}, y_{3}\right) & =w_{k}\left(y_{1}, y_{2}, y_{3}\right), \\
w_{k}\left(y_{1}+1 / 2, y_{2}+h_{\mathrm{p}}, y_{3}\right) & =w_{k}\left(y_{1}, y_{2}, y_{3}\right), \\
w_{k}\left(y_{1}+1 / 2, y_{2}, y_{3}+h_{\mathrm{p}}\right) & =w_{k}\left(y_{1}, y_{2}, y_{3}\right),
\end{aligned}
$$

where

$$
\begin{aligned}
& -\Delta w_{k}(\vec{y})=0 \quad \text { in }[0,1] \times\left[0, h_{\mathrm{c}}\right] \times\left[0, h_{\mathrm{c}}\right], \\
& -\partial_{y_{1}} w_{k}\left(1, y_{2}, y_{3}\right)-\kappa_{\text {end }}\left(w_{k}\left(1, y_{2}, y_{3}\right)-w_{k}\left(0, y_{2}, y_{3}\right)\right)=\delta_{k 1}, \\
& -\partial_{y_{2}} w_{k}\left(y_{1}, h_{\mathrm{c}}, y_{3}\right)-\kappa_{\text {side }}\left(w_{k}\left(y_{1}, h_{\mathrm{c}}, y_{3}\right)-w_{k}\left(y_{1}+1 / 2,0, y_{3}\right)\right)=\delta_{k 2}, \\
& -\partial_{y_{3}} w_{k}\left(y_{1}, y_{2}, h_{\mathrm{c}}\right)-\kappa_{\text {side }}\left(w_{k}\left(y_{1}, y_{2}, h_{\mathrm{c}}\right)-w_{k}\left(y_{1}+1 / 2, y_{2}, 0\right)\right)=\delta_{k 3}, \\
& -\partial_{y_{1}} w_{k}\left(1, y_{2}, y_{3}\right)=-\partial_{y_{1}} w_{k}\left(0, y_{2}, y_{3}\right), \\
& -\partial_{y_{2}} w_{k}\left(y_{1}, h_{\mathrm{c}}, y_{3}\right)=-\partial_{y_{2}} w_{k}\left(y_{1}, 0, y_{3}\right), \\
& -\partial_{y_{3}} w_{k}\left(y_{1}, y_{2}, h_{\mathrm{c}}\right)=-\partial_{y_{3}} w_{k}\left(y_{1}, y_{2}, 0\right) .
\end{aligned}
$$

The procedure for computing effective conductivities with a brick-like arrangement is slightly different than that for the uniform arrangement. As a cross-section normal to the $x_{1}$-direction intersects cells at two different values of the local $y_{1}$ coordinate, intracellular longitudinal conductivity is now

$$
\sigma_{\mathrm{i}, \mathrm{l}}=\frac{\sigma_{\mathrm{c}}}{2 h_{\mathrm{p}}^{2}} \int_{0}^{h_{\mathrm{c}}} \int_{0}^{h_{\mathrm{c}}}\left(2+\partial_{y_{1}} w_{1}\left(y_{1}, y_{2}, y_{3}\right)+\partial_{y_{1}} w_{1}\left(y_{1}+1 / 2, y_{2}, y_{3}\right)\right) d y_{2} d y_{3} .
$$

The solutions to (32)-(38) for $w_{2}$ and $w_{3}$ in the brick-like arrangement are identical to those of (16)-(22) in the aligned arrangement. Hence, formula (29) holds for the computation of $\sigma_{\mathrm{i}, \mathrm{t}}$ in the brick-like cellular arrangement.

\subsection{Resulting effective conductivities}

We solve (32)-(38) numerically, using a standard second-order accurate 7-point finite difference discretization of the Laplacian on a $100 \times 10 \times 10$ cell-centered grid. We enforce Neumann boundary conditions by introducing ghost points and solve the resulting linear system by Gaussian elimination. Using numerical values in Table B.1, the effective intracellular conductivities are

$$
\begin{aligned}
\sigma_{\mathrm{i}, 1} & =1.40 \mathrm{mS} / \mathrm{cm}, \\
\sigma_{\mathrm{i}, \mathrm{t}} & =0.034 \mathrm{mS} / \mathrm{cm} .
\end{aligned}
$$

The numerical value of $\sigma_{\mathrm{i}, 1}$ agrees with that obtained on a $200 \times 20 \times 20$ grid up to five digits of accuracy. The value for $\sigma_{\mathrm{i}, \mathrm{t}}$ is the same as formula (31), obtained from (29). 
Figure 6 shows the response in intracellular potential to a voltage applied to cells in a brick-like arrangement.

To give a sense for the conductivities that can arise from aligned and brick-like arrangements, Table 1 provides the values for $\sigma_{\mathrm{i}, 1}$ computed for various values of $g_{\mathrm{GJ}, \text { end }}$ and $g_{\mathrm{GJ}, \text { side }}$.

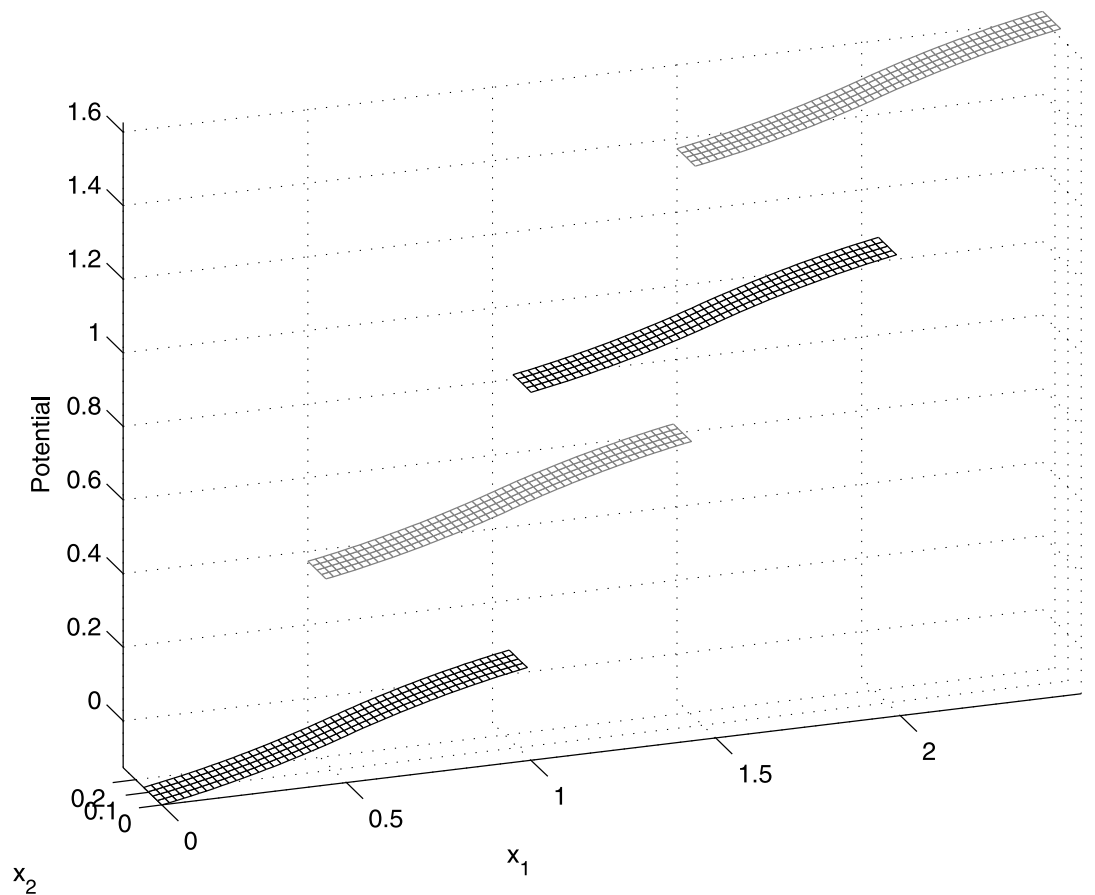

Fig. 6 Electric potential in several cells in a brick-like arrangement with a longitudinally applied potential gradient using cellular parameters from Table B.1. The second and fourth cells from the left are offset by half a cell length because they are in a neighboring fiber to the first and third cells. The units of the vertical axis are arbitrary. As the cellular domain is three dimensional, the plot shows only the $x_{3}$-averaged potential within a cell.

Table 1 Intracellular, longitudinal conductivities $\sigma_{\mathrm{i}, 1}(\mathrm{mS} / \mathrm{cm})$ obtained from the aligned and brick-like arrangements for various values of $g_{\mathrm{GJ}, \text { end }}$ and $g_{\mathrm{GJ}}$,side. The numbers before the commas are for the aligned arrangement. The numbers after the commas are for the brick-like arrangement. Note that the conductivities for the brick-like arrangement depend on $g_{\mathrm{GJ}}$, side, but those for the aligned arrangement do not

\begin{tabular}{lllll}
\hline$\sigma_{\mathrm{i}, 1}$ & & $g_{\mathrm{GJ}, \text { side }}$ & & \\
\cline { 3 - 5 } & & & & \\
\hline$g_{\mathrm{GJ}, \text { end }}$ & $2.94 \times 10^{-4} \mathrm{mS}$ & $5.88 \times 10^{-4} \mathrm{mS}$ & $1.18 \times 10^{-3} \mathrm{mS}$ \\
& $5.58 \times 10^{-4} \mathrm{mS}$ & $0.55,0.80$ & $0.55,1.02$ & $0.55,1.38$ \\
& $1.12 \times 10^{-3} \mathrm{mS}$ & $1.01,1.22$ & $1.01,1.40$ & $1.01,1.70$ \\
& $1.72,1.87$ & $1.72,2.00$ & $1.72,2.23$ \\
\hline
\end{tabular}


Table 2 Conductivity values obtained by fitting macroscopic wavespeed data to solutions of bidomain equations. Values are obtained for ventricular tissue from various animals, such as dogs, cows, and sheep

\begin{tabular}{lll}
\hline Conductivity type & Value $(\mathrm{mS} / \mathrm{cm})$ & Reference \\
\hline$\sigma_{\mathrm{i}, 1}$ & 1.6 & Weidmann (1970) \\
& 1.7 & Clerc (1976) \\
& 2.8 & Roberts et al. (1979) \\
& 3.4 & Roberts and Scher (1982) \\
& 4.5 & Kléber and Riegger (1987) \\
& 0.2 & Clerc (1976) \\
$\sigma_{\mathrm{i}, \mathrm{t}}$ & 0.3 & Roberts et al. (1979) \\
& 0.6 & Roberts and Scher (1982) \\
& 1.2 & Roberts and Scher (1982) \\
$\sigma_{\mathrm{e}, 1}$ & 2.2 & Roberts et al. (1979) \\
& 4.0 & Kléber and Riegger (1987) \\
& 5.3 & Weidmann (1970) \\
& 6.3 & Clerc (1976) \\
$\sigma_{\mathrm{e}, \mathrm{t}}$ & 0.8 & Roberts and Scher (1982) \\
& 1.3 & Roberts et al. (1979) \\
& 2.4 & Clerc (1976) \\
\hline
\end{tabular}

\section{Discussion}

We remark that our solutions to Laplace's equation in the aligned and brick-like arrangements have the saw-tooth nature that has been characteristic of many studies in modeling cardiac tissue (Neu and Krassowska, 1993; Krassowska et al., 1990; Keener, 1996). In the aligned arrangement with potential applied in one of the principal directions, the profile is linear in cells, as given by the corrector functions (25)-(27). In the brick-like arrangement, the solution shown in Fig. 6 has a piecewise nonlinear shape due to the current through the side-to-side gap junctions, even though potential is only applied longitudinally. We remark that the primary difference between our model and those of Neu and Krassowska (1993) and Krassowska et al. (1990) is that we model gap junctions through boundary conditions, and not as discrete geometrical entities. Recall Fig. 1.

\subsection{Effective conductivity values}

Table 2 presents values from the literature for effective conductivities obtained by fitting macroscopic measurements of conduction velocity to solutions of the cable or bidomain equations. Table 3 presents the corresponding values of our calculations, obtained from microscopic measurements via homogenization. Our values for $\sigma_{\mathrm{i}, 1}, \sigma_{\mathrm{e}, 1}$, and $\sigma_{\mathrm{e}, \mathrm{t}}$ lie within the reported ranges in Table 2. Our value for $\sigma_{\mathrm{i}, \mathrm{t}}$, however, is about an order of magnitude smaller than the values from this literature based on macroscopic measurements. 
Table 3 Conductivity values obtained directly from microscopic measurements via homogenization in the present work

\begin{tabular}{llll}
\hline Conductivity type & Value $(\mathrm{mS} / \mathrm{cm})$ & Arrangement & Equation \\
\hline$\sigma_{\mathrm{i}, 1}$ & 1.01 & Aligned & $(30)$ \\
& 1.40 & Brick-like & $(40)$ \\
$\sigma_{\mathrm{i}, \mathrm{t}}$ & 0.03 & Aligned or brick-like & $(31)$ \\
$\sigma_{\mathrm{e}, \mathrm{l}}$ & 3.00 & Aligned or brick-like & (A.8) \\
$\sigma_{\mathrm{e}, \mathrm{t}}$ & 1.56 & Aligned or brick-like & (A.9) \\
\hline
\end{tabular}

Our calculation of intracellular and extracellular effective conductivities compare favorably to those obtained by Stinstra et al. (2005) using a much more detailed cellular architecture. Our values for $\sigma_{\mathrm{i}, 1}, \sigma_{\mathrm{i}, \mathrm{t}}, \sigma_{\mathrm{e}, \mathrm{l}}$ and $\sigma_{\mathrm{e}, \mathrm{t}}$ are within a factor of 3 of those of Stinstra et al. Such differences should be expected as Stinstra et al. use different values for myocyte dimensions, conductivities, and other microscopic properties.

We will now explore several possible explanations of the discrepancy in transverse conductivities between calculations based in macroscopic and microscopic measurements. Stinstra et al. propose two possible explanations for the discrepancy in $\sigma_{\mathrm{i}, \mathrm{t}}$. These are: (1) the total measured gap junctional conductivity of single cells may be too low, and (2) the measured fraction of gap junctions located on the sides of myocytes may be too low.

To address the first possible explanation, we use (29) to ask what the microscopic measurement of $g_{\mathrm{GJ}, \text { side }}$ would have to be in order for the effective transverse intracellular conductivity to be in the range reported in Table 2 . For a typical value like $\sigma_{\mathrm{i}, \mathrm{t}}=0.2 \mathrm{mS} / \mathrm{cm}$, the microscopic measurement of $g_{\mathrm{GJ}}$,side would have to be $3.5 \times 10^{-3} \mathrm{mS} / \mathrm{cm}$, which is an order of magnitude larger than the value reported in Yao et al. (2003).

To address the second possible explanation, we study the effects of gap junctional distribution on transverse extracellular conductivity. We assume that the total conductance of all gap junctions within a cell is $4 \times g_{\mathrm{GJ}, \text { side }}+2 \times g_{\mathrm{GJ}, \text { end }}$. If all this conductance is localized exclusively to the sides of each cell, $\sigma_{\mathrm{i}, \mathrm{t}}$ becomes $5.0 \times 10^{-2} \mathrm{mS} / \mathrm{cm}$, which is still much smaller than the range of values reported in Table 2. Thus, gap junctional distribution is not the primary explanation of the discrepancy between $\sigma_{\mathrm{i}, \mathrm{t}}$ calculated from microscopic parameters and the values from experimental measurements.

An additional possibility is that our microscopic model omits an important cellular mechanism that aides transverse conductivity. Two such mechanisms include ephaptic propagation (Mori et al., 2008) and electrical coupling via gap junctions between myocytes and fibroblasts (Kohl et al., 2005; Camelliti et al., 2006). We leave the incorporation of such mechanisms into our model as future work.

As noted in Neu and Krassowska (1993), another possible explanation of the discrepancy is that the experimental measurements are unable to completely distinguish longitudinal and transverse conductivity. For example, even though Roberts et al. (1979) performed in situ surface recordings, the propagation pattern is influenced by transmural fiber rotation. The inferred value of intracellular transverse conductivity will therefore be an overestimate.

Our model of cellular architecture allows us to compare the effects of a brick-like cellular arrangement on intracellular conductivities. As mentioned in Section 5, the change 

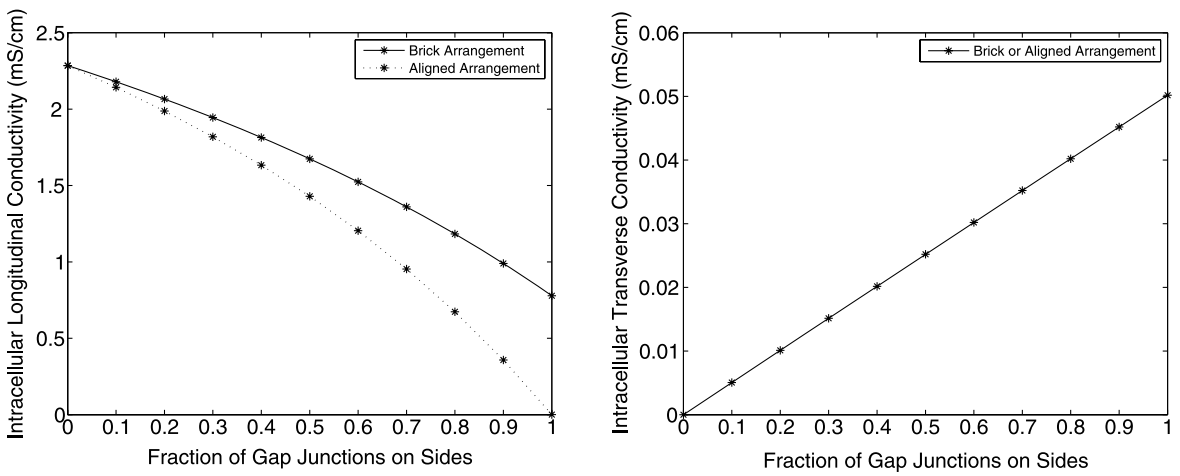

Fig. 7 Effective intracellular transverse and longitudinal conductivities as a function of the fraction of gap junctions expressed on the cell sides, keeping the total conductance constant. As gap junctions are moved from the ends to the sides of a cell, intracellular longitudinal conductivity decreases (a). With an aligned arrangement it decreases to zero, but with the brick arrangement there is a nonzero intracellular longitudinal conductivity even when all gap junctions are on the sides of the cells. Meanwhile, in the parameter regime given by cellular measurements, intracellular transverse conductivity increases linearly with the fraction of gap junctions on the cell sides (b). The side-to-side and end-to-end conductance measurements given in Table B. 1 correspond to a fraction 0.68 of gap junctions on the sides of cells. A uniform density of gap junctions between the sides and ends of cells with dimensions given in Table B.1 corresponds to a fraction 0.92 of gap junctions on the sides of cells.

from an aligned architecture to a brick-like one does not influence the transverse intracellular conductivity under our model. Comparing (40) to (30), we see that a brick-like arrangement has $40 \%$ more intracellular longitudinal conductivity than the corresponding aligned arrangement. Note that this increase is parameter-dependent; specifically, it depends on the distribution of gap junctions between the ends and the sides of the cells. As shown in Fig. 7, if all the gap junctions are located on the sides of the cells, the intracellular longitudinal conductivity is zero in the aligned architecture but is non-zero in the brick-like architecture. If all gap junctions are located on the ends of the cells, there is no transverse conductivity and no difference between the brick-like and aligned arrangements. A brick-like arrangement of cells allows side-to-side coupling to contribute to both longitudinal and transverse conductivity. In an aligned arrangement of cells, however, side-to-side coupling contributes only to transverse conductivity.

\subsection{Comparison of PDE and resistor network methods}

Resistor network models of cardiac tissue sometimes allows analytical computations of conductivity that are more elementary than those from PDE models. The calculation in Section 4.3 and the hexagonal network from Neu and Krassowska (1993) are both examples. To be useful, the PDE approach should provide some benefit. This benefit is that it allows one to investigate effects of different cell shapes, cell arrangements, and distributions of gap junctions within the ends and sides of cells. The simple equivalent resistor network from Section 4.3 is only possible because of the one-dimensional nature of solutions to Laplace's equation in the aligned arrangement when voltage is applied along one of the principal directions. 
Alternate cell shapes, arrangements, and gap junction distributions introduce a nontrivial dependence on multiple spatial dimensions and would make equivalent resistor networks complicated at best. One way to obtain such a network, albeit only approximately, would be to introduce a discretization of Laplace's equation (8)-(14). To be accurate, it would need to involve many resistors per cell. Possibly, the corrector functions for the brick-like arrangement could be adequately and analytically computed under a very coarse grid, but we do not pursue this idea here.

\subsection{Application to electromechanical simulations}

A goal of this work is to strengthen the connection between measured cellular parameters and the effective conductivities used in whole-heart bidomain simulations. This connection may be particularly useful in the context of simulations coupling cardiac electrophysiology with muscle mechanics (Panfilov et al., 2007). As a region of cardiac muscle contracts, the length, width, and cross-sectional area fraction of cells will vary. Because the effective conductivities depend on these parameters, they may need to be recalculated at each time step in such a simulation. In Panfilov et al. (2007), the authors demonstrate nontrivial electro-mechanical interactions in the setting of fixed macroscopic conductivity tensors. Similar simulations may reveal new interactions resulting from deformationdependent conductivities.

One complication in recomputing conductivities based on deformation is that volume preserving contractions of rectangular prisms do not preserve surface area. As a result, cardiac myocyte membranes presumably wrinkle during contraction. Under our model, we could view a rectangularly shaped cell with surface wrinkles as a perfect rectangular prism with a different gap junctional density. Our model accounts for this change in density automatically because it would distribute the prescribed conductance of gap junctions over the ends and sides of the deformed rectangular prism. Thus, our approach for computing effective conductivity tensors is well suited for bidomain simulations which include muscle deformation.

\section{Conclusion}

This paper attempts to find the right level of detail of cyto-architecture and gap junctional connectivity in order to produce effective conductivity values for bidomain simulations based on directly measurable quantities. We propose that this right level of detail is obtained by modeling cells as simple prisms and gap junctions as continuous Neumann boundary conditions to Laplace's equation.

The strengths of our approach are that (1) the physical parameters it requires are directly measurable; (2) it distinguishes end-to-end and side-to-side conductivities, which can be separately measured; and (3) it is a mathematically natural framework within which to view the effects of cell arrangements, geometrical parameters, and the distribution of gap junctions within cells. For the cases where it is not possible to obtain analytical solutions, numerical solutions are obtained by solving Laplace's equation on simple domains and with standard boundary conditions.

The ease of computation afforded by our approach makes it attractive for use as part of an electromechanical simulation. As cardiac muscle deforms, the effective conductivity 
tensors change and would need to be recalculated at each timestep. For such purposes, it is important to have inexpensive calculations, such as analytical formulae or solutions to Laplace's equation on simple domains.

A weakness of our approach is its inability to deal with random variation in cytoarchitecture. Although the corrector problem could be phrased over a domain consisting of several randomly generated cells, there would be little benefit to the homogenization point of view. The simulations of Stinstra et al. (2005) indicate that variability in cellular properties has a relatively small impact on macroscopic conductivities. Consequently, the inability to handle randomness does not severely limit the applicability of our approach. Although, the levels of complexity of our model and that of Stinstra et al. (2005) are quite different, the effective conductivity values they predict are quite similar.

\section{Acknowledgements}

We gratefully acknowledge discussions with Glenn Fishman and Greg Morley at NYU School of Medicine. We also wish to express our appreciation to Professor Fishman for early access to experimental measurements of the cellular dimensions of mouse ventricular myocytes. We thank an anonymous reviewer for insightful comments pertaining to equivalent resistor networks. P.H. was supported by the Department of Defense through a National Defense Science and Engineering Graduate Fellowship.

\section{Appendix A: Extracellular conductivities}

In our model of myocardial geometry, the extracellular domain $\Omega_{\mathrm{e}}$ is translationally symmetric in the longitudinal direction but is periodically perforated in the transverse directions. Figure A.1 shows an $x_{2}, x_{3}$ cross-section of it. Extracellular potential satisfies Laplace's equation in $\Omega_{\mathrm{e}}$ with no-flux boundary conditions. Hence, effective extracellular longitudinal conductivity is found by solving

$$
\begin{aligned}
\Delta \phi_{\mathrm{e}}(\vec{x}) & =0 \quad \text { in } \Omega_{\mathrm{e}}, \\
\phi_{\mathrm{e}}\left(0, x_{2}, x_{3}\right) & =0, \\
\phi_{\mathrm{e}}\left(1, x_{2}, x_{3}\right) & =V, \\
\partial_{x_{2}} \phi_{\mathrm{e}}\left(x_{1}, 0, x_{3}\right) & =0, \\
\partial_{x_{2}} \phi_{\mathrm{e}}\left(x_{1}, 1, x_{3}\right) & =0, \\
\partial_{x_{3}} \phi_{\mathrm{e}}\left(x_{1}, x_{2}, 0\right) & =0, \\
\partial_{x_{3}} \phi_{\mathrm{e}}\left(x_{1}, x_{2}, 1\right) & =0,
\end{aligned}
$$

and computing

$$
\sigma_{\mathrm{e}, 1}=\sigma_{\mathrm{ext}} \frac{V}{\frac{1}{A} \iint_{\Omega_{\mathrm{e}} \cap\left\{x_{1}=c\right\}} \partial_{x_{1}} \phi_{\mathrm{i}} d x_{2} d x_{3}},
$$




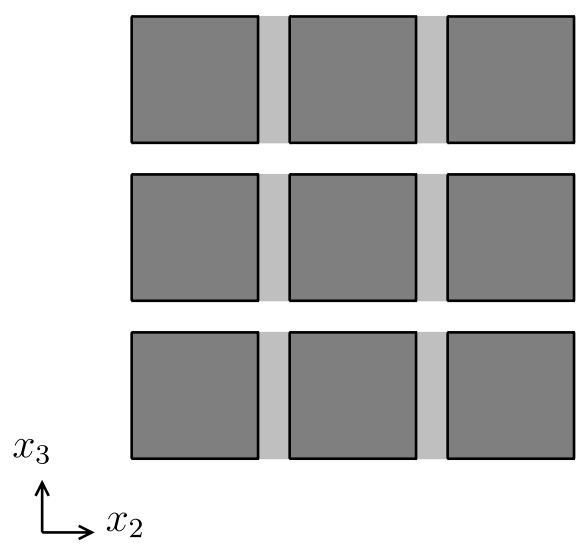

Fig. A.1 In this cross-section of tissue, the longitudinal direction points out of the page. The dark shaded regions indicate cells, and the remaining regions indicate extracellular space. We treat the light shaded regions as insulators for the calculation of transverse conductivity in the $x_{2}$-direction.

where $A$ is the cross-sectional area of the tissue, the domain of integration is the set of exterior points of a cross-section, and the integral is independent of the constant $c$.

The extracellular transverse conductivity can be found by altering the boundary conditions in (A.1)-(A.7) so that voltage is imposed and current is measured in the $x_{2}$-direction.

The response to a longitudinally applied potential gradient is linear, and hence the effective conductivity is the extracellular conductivity scaled by the cross-sectional area fraction of extracellular space $\alpha$.

In the case that $\alpha$ is small, the response to a transversely applied potential gradient in the $x_{2}$ direction is approximately linear because of the high resistance of the regions indicated with light shading in Fig. A.1. In that case, the extracellular conductivity is scaled by the area fraction of extracellular space in the transverse direction, $1-\sqrt{1-\alpha}$. This rough calculation agrees up to $O\left(\alpha^{2}\right)$ with the derivation in Neu and Krassowska (1993).

With the values of parameters given in Table B.1, the extracellular longitudinal and transverse conductivities are

$$
\begin{aligned}
& \sigma_{\mathrm{e}, \mathrm{l}}=\sigma_{\mathrm{ext}} \alpha=3.00 \mathrm{mS} / \mathrm{cm}, \\
& \sigma_{\mathrm{e}, \mathrm{t}}=\sigma_{\mathrm{ext}}(1-\sqrt{1-\alpha})=1.56 \mathrm{mS} / \mathrm{cm} .
\end{aligned}
$$

Alternatively, $\sigma_{\mathrm{e}, \mathrm{t}}$ could have been computed by homogenization. The extracellular domain $\Omega_{\mathrm{e}}$ is periodic, and the extracellular potential satisfies Laplace's equation with noflux boundary conditions on the cell membranes. The calculation would proceed exactly as in Section 4 but with the corrector

$$
\begin{aligned}
\Delta_{y} w_{k}(x ; y) & =0 \quad \text { in } Y_{\mathrm{e}}, \\
-\partial_{\nu} w_{k} & =e_{k} \cdot v \quad \text { on } \partial Y_{\mathrm{e}},
\end{aligned}
$$

where $Y_{\mathrm{e}}$ is the periodic unit of the extracellular space depicted in Fig. 4, $e_{k}$ is the unit vector in the $x_{k}$ direction, and $v$ is the unit outer normal vector to the extracellular space. 


\section{Appendix B: Parameters and variables}

Table B.1 The physical parameters, derived parameters, and variables that enter our cellular model. The measured parameters of Yao et al. (2003) and Fishman (2008) correspond to mouse ventricular myocytes

\begin{tabular}{|c|c|c|c|}
\hline Symbol & Parameter & Value & Reference \\
\hline$\sigma_{\mathrm{c}}$ & Conductivity of cytoplasm & $6.7 \mathrm{mS} / \mathrm{cm}$ & Shaw and Rudy (1997) \\
\hline$\sigma_{\mathrm{ext}}$ & Conductivity of extracellular space & $20 \mathrm{mS} / \mathrm{cm}$ & Neu and Krassowska (1993) \\
\hline$g_{\mathrm{GJ}, \text { end }}$ & $\begin{array}{l}\text { Total conductance of all gap } \\
\text { junctions on one end of cell }\end{array}$ & $5.58 \times 10^{-4} \mathrm{mS}$ & Yao et al. (2003) \\
\hline$g_{\mathrm{GJ}, \text { side }}$ & $\begin{array}{l}\text { Total conductance of all gap } \\
\text { junctions one side of cell }\end{array}$ & $5.88 \times 10^{-4} \mathrm{mS}$ & Yao et al. (2003) \\
\hline$l$ & Length of cell & $1.58 \times 10^{-2} \mathrm{~cm}$ & Fishman (2008) \\
\hline$w_{\mathrm{c}}$ & Width of cell & $2.47 \times 10^{-3} \mathrm{~cm}$ & Fishman (2008) \\
\hline$\alpha$ & Volume fraction of extracellular space & 0.15 & Neu and Krassowska (1993) \\
\hline \multicolumn{4}{|c|}{ Derived parameters and variables } \\
\hline Symbol & Parameter & Value & \\
\hline$w_{\mathrm{p}}$ & Transverse period & $\frac{w_{\mathrm{c}}}{\sqrt{1-\alpha}}$ & \\
\hline$h_{\mathrm{c}}$ & Aspect ratio of cells & $\frac{w_{c}}{l}$ & \\
\hline$h_{\mathrm{p}}$ & Aspect ratio of period & $\frac{w_{\mathrm{p}}}{l}$ & \\
\hline$\kappa_{\text {end }}$ & $\begin{array}{l}\text { Nondimensional parameter } \\
\text { of total end-to-end gap } \\
\text { junctional conductance }\end{array}$ & $\frac{g_{\mathrm{GJ}, \text { end }} l}{\sigma_{\mathrm{c}} w_{\mathrm{c}}^{2}}$ & \\
\hline$\kappa_{\text {side }}$ & $\begin{array}{l}\text { Nondimensional parameter } \\
\text { of total side-to-side gap } \\
\text { junctional conductance }\end{array}$ & $\frac{g_{\mathrm{GJ}, \text { side }}}{\sigma_{\mathrm{c}} w_{\mathrm{c}}}$ & \\
\hline$\vec{x}$ & Macroscale spatial coordinates & & \\
\hline$\vec{y}$ & Microscale spatial coordinates & & \\
\hline$\varepsilon$ & $\begin{array}{l}\text { Nondimensional cell length } \\
\text { relative to significant electric } \\
\text { potential variations in the tissue }\end{array}$ & & \\
\hline
\end{tabular}

\section{References}

Camelliti, P., Green, C., Kohl, P., 2006. Structural and functional coupling of cardiac myocytes and fibroblasts. In: Dhein, S. (Ed.), Cardiovascular Gap Junctions, Adv. Cardiol., vol. 42, pp. 132-149. Karger, Basel. 
Clerc, L., 1976. Directional differences of impulse spread in trabecular muscle from mammalian heart. J. Physiol. 255, 335-346.

Fishman, G., 2008. Personal communication.

Henriquez, C., 1993. Simulating the electrical behavior of cardiac tissue using the bidomain model. Crit. Rev. Biomed. Eng. 21, 1-77.

Hubbard, M., Ying, W., Henriquez, C., 2007. Effect of gap junction distribution on impulse propagation in a monolayer of myocytes: a model study. Europace 9, vi20-vi28.

Keener, J., 1996. Direct activation and defibrillation of cardiac tissue. J. Theor. Biol. 178, 313-324.

Keener, J., Sneyd, J., 1998. Mathematical Physiology. Springer, Berlin.

Kléber, A., Riegger, C., 1987. Electrical constants of arterially perfused rabbit papillary muscle. J. Physiol. 385, 307-324.

Kohl, P., Camelliti, P., Vurton, F., Smith, G., 2005. Electrical coupling of fibroblasts and myocytes: relevance for cardiac propagation. J. Electrocardiol. 38, 45-50.

Krassowska, W., Pilkington, T., Ideker, R., 1990. Potential distribution in three-dimensional periodic myocardium-Part I: solution with two-scale asymptotic analysis. IEEE Trans. Biomed. Eng. 37(3), 252-266.

Mori, Y., Fishman, G., Peskin, C., 2008. Ephaptic conduction in a cardiac strand model with 3D eletrodiffusion. Proc. Natl. Acad. Sci. 105, 6463-6468.

Neu, J., Krassowska, W., 1993. Homogenization of syncytial tissues. Crit. Rev. Biomed. Eng. 21, $137-199$.

Panfilov, A., Keldermann, R., Nash, M., 2007. Drift and breakup of spiral waves in reaction-diffusionmechanics systems. Proc. Natl. Acad. Sci. 104, 7922-7926.

Roberts, D., Scher, A., 1982. Effect of tissue anisotropy on extracellular potential fields in canine myocardium in situ. Circ. Res. 50, 342-351.

Roberts, D., Hersh, L., Scher, A., 1979. Influence of cardiac fiber orientation on wavefront voltage, conduction velocity, and tissue resistivity in the dog. Circ. Res. 44, 701-712.

Shaw, R., Rudy, Y., 1997. Ionic mechanisms of propagation in cardiac tissue-roles of the sodium and L-type calcium currents during reduced excitability and decreased gap junction coupling. Circ. Res. $81,727-741$.

Stinstra, J., Hopenfeld, B., Macleod, R., 2005. On the passive cardiac conductivity. Ann. Biomed. Eng. 33, 1743-1751.

Weidmann, S., 1970. Electrical constants of trabecular muscle from mammalian heart. J. Physiol. 210, 1041-1054.

Yao, J., Gutstein, D., Liu, F., Fishman, G., Wit, A., 2003. Cell coupling between ventricular myocyte pairs from connexin43-deficient murine hearts. Circ. Res. 93, 736-743. 